\title{
Monotone and second order consistent scheme for the two dimensional Pucci equation
}

\author{
Joseph Frédéric Bonnans, Guillaume Bonnet ${ }^{\dagger}$, Jean-Marie Mirebeau ${ }^{\ddagger}$
}

May 31, 2020

\begin{abstract}
We introduce a new strategy for the design of second-order accurate discretizations of non-linear second order operators of Bellman type, which preserves degenerate ellipticity. The approach relies on Selling's formula, a tool from lattice geometry, and is applied to the Pucci equation, discretized on a two dimensional Cartesian grid. Numerical experiments illustrate the robustness and the accuracy of the method.
\end{abstract}

\section{Introduction}

Degenerate Ellipticity (DE) is a property of a class of partial differential operators, often non-linear and of order at most two. When satisfied, it implies a generalized comparison principle, from which can be deduced the existence, uniqueness and stability of a viscosity solution to the Partial Differential Equation (PDE), under mild additional assumptions [CIL92]. Discrete degenerate ellipticity is the corresponding property for numerical schemes, see Definition 2.3, which has similarly strong implications and often turns the convergence analysis of solutions into a simple verification [Obe06]. It is therefore appealing to design PDE discretizations preserving the DE property, yet a strong limitation of the current approaches [BS91, Obe08, FJ17] is their low consistency order, usually below one. Filtered schemes [FO13] attempt to mitigate this issue by combining a DE scheme of low consistency order with a non-DE scheme of high consistency order, but their use requires careful parameter tuning, and theoretical results are lacking regarding their effective accuracy.

In this paper, we propose a new approach to develop second order accurate DE schemes, which is the highest achievable consistency order Obe06], on two dimensional Cartesian grids. The operator must be given in Bellman form as follows

$$
\Lambda u(x)=\sup _{\alpha \in \mathcal{A}} a_{\alpha}+b_{\alpha} u(x)-\operatorname{Tr}\left(D_{\alpha} \nabla^{2} u(x)\right),
$$

*INRIA Saclay, Ecole Polytechnique, CMAP 91128 Palaiseau

${ }^{\dagger}$ U. Paris-Sud, U. Paris-Saclay, 91405, Orsay, France

$¥$ U. Paris-Sud, CNRS, U. Paris-Saclay, 91405, Orsay

This work was partly supported by ANR research grant MAGA, ANR-16-CE40-0014 The first author acknowledges support from the FIME laboratory, and from the Labex Mathématiques Hadamard and iCODE (IDEX Paris-Saclay) for the PGMO project "Optimal control of conservation equations". 
where $\mathcal{A}$ is an abstract set of parameters, and the coefficients $a_{\alpha} \in \mathbb{R}, b_{\alpha} \geq 0$, and symmetric positive definite matrix $D_{\alpha}$ may additionally depend on the position $x$. A specific feature of our approach, that is tied to the structure of the addressed problems, is that the parameter space $\mathcal{A}$ is not discretized. We apply this approach to the two dimensional Pucci equation:

$$
\lambda_{\min }\left(\nabla^{2} u(x)\right)+\mu \lambda_{\max }\left(\nabla^{2} u(x)\right)=f(x),
$$

with Dirichlet boundary conditions, where $\lambda_{\min }$ and $\lambda_{\max }$ denote the smallest and largest eigenvalue of a symmetric matrix, and where $\mu>0$. This PDE admits the following Bellman form, when $\mu \leq 1$, which we assume for simplicity:

$$
\max _{\theta \in[0, \pi]}-\operatorname{Tr}\left(D(\theta, \mu) \nabla^{2} u(x)\right)=-f(x), \quad \text { where } D(\theta, \mu):=R_{\theta}\left(\begin{array}{ll}
1 & 0 \\
0 & \mu
\end{array}\right) R_{\theta}^{T},
$$

and where $R_{\theta}:=\left(\begin{array}{cc}\cos \theta & -\sin \theta \\ \sin \theta & \cos \theta\end{array}\right)$ denotes the rotation matrix of angle $\theta \in \mathbb{R}$. Our approach also applies in the case $\mu \geq 1$, with only the slight modification that the max in (3) is replaced with a min. Note that the optimization space in (3) is $\mathcal{A}=[0, \pi]$, which is compact and one dimensional, thus easing the theoretical study and the numerical implementation.

Motivation for this study. The Pucci equation interpolates between two fundamental problems in analysis: the Poisson problem when $\mu=1$, and the (lower)convex envelope of the boundary conditions when $\mu=0$ and $f=0$. It is also an excellent representative of the class of Pucci extremal operators, a.k.a. operators which can be written in the form (1), perhaps replacing the inf with a sup. This class also encompasses the Monge-Ampère operator, known for its applications in optimal transport and optics, to which similar techniques may be applied [BCM16].

\section{Discretization}

We rely on a tool from algorithmic lattice geometry, known as Selling's formula \$2.1. which is particularly adequate for discretizing degenerate elliptic PDEs on Cartesian grids of dimension two BOZ04 or three [Mir18, Mir19, FM14. Throughout this section $\Omega \subset \mathbb{R}^{2}$ denotes a bounded domain, and $h>0$ a grid scale. Define

$$
\Omega_{h}:=h \mathbb{Z}^{2} \cap \Omega, \quad \Delta_{h}^{e} u(x):=\frac{u(x+h e)-2 u(x)+u(x-h e)}{h^{2}},
$$

the discrete domain and the second order finite difference of a map $u: \Omega_{h} \cup \partial \Omega \rightarrow \mathbb{R}$ at $x \in \Omega_{h}$ in the direction $e \in \mathbb{Z}^{2}$. When $x$ is adjacent to $\partial \Omega$ the latter formula becomes

$$
\Delta_{h}^{e} u(x):=\frac{2}{h_{+}+h_{-}}\left(\frac{u\left(x+h_{+} e\right)-u(x)}{h_{+}}+\frac{u\left(x-h_{-} e\right)-u(x)}{h_{-}}\right),
$$

where $h_{ \pm}>0$ is the least value such that $x \pm h_{ \pm} e \in \Omega_{h} \cup \partial \Omega$. Note that (4, right) is a second order consistent approximation of $\left\langle e, \nabla^{2} u(x) e\right\rangle$, whereas (5) is only first order consistent. Thus

$$
\operatorname{Tr}\left(e e^{T} \nabla^{2} u(x)\right)=\left\langle e, \nabla^{2} u(x) e\right\rangle=\Delta_{h}^{e} u(x)+\mathcal{O}\left(h^{r}\right),
$$

where $r=1$ if $x$ is adjacent to $\partial \Omega$, and $r=2$ otherwise. 


\subsection{Selling's formula}

Selling's decomposition of an element of the set $S_{2}^{++}$of symmetric positive definite $2 \times 2$ matrices, see Proposition 2.2. can be regarded as a variant of the eigenvector/eigenvalue decomposition, but whose vectors have integer entries. We rely on it to discretize non-divergence form linear (8) and non-linear (11) operators, in a manner that achieves discrete degenerate ellipticity, see Definition 2.3 .

Definition 2.1. A superbase of $\mathbb{Z}^{2}$ is a triplet $\left(e_{0}, e_{1}, e_{2}\right) \in\left(\mathbb{Z}^{2}\right)^{3}$ such that $e_{0}+$ $e_{1}+e_{2}=0$ and $\left|\operatorname{det}\left(e_{1}, e_{2}\right)\right|=1$. It is D-obtuse, where $D \in S_{2}^{++}$, iff $\left\langle e_{i}, D e_{j}\right\rangle \leq 0$ for all $i \neq j$.

Proposition 2.2 (Selling [Sel74]). For each $D \in S_{2}^{++}$there exists a D-obtuse superbase $\left(e_{0}, e_{1}, e_{2}\right)$ of $\mathbb{Z}^{2}$, which can be obtained from Selling's algorithm. Furthermore one has Selling's formula

$$
D=\sum_{0 \leq i \leq 2} \rho_{i} v_{i} v_{i}^{\top} \quad \text { with } \rho_{i}:=-\left\langle e_{i-1}, D e_{i+1}\right\rangle \geq 0, \quad v_{i}:=e_{i}^{\perp} \in \mathbb{Z}^{2},
$$

where $e^{\perp}:=(-b, a)^{\top}$ if $e=(a, b)^{\top} \in \mathbb{R}^{2}$. The set $\left\{\left(\rho_{i}, \pm v_{i}\right) ; 0 \leq i \leq 2, \rho_{i}>0\right\}$ is uniquely determined. In (7), the indices $i-1$ and $i+1$ are understood modulo 3 .

Based on this formula, one can consider the following finite differences operator:

$$
\Delta_{h}^{D} u(x):=\sum_{0 \leq i \leq 2} \rho_{i} \Delta_{h}^{v_{i}} u(x) .
$$

Using (6), (7), (8) and the linearity of the trace operator on matrices, we obtain

$$
\operatorname{Tr}\left(D \nabla^{2} u(x)\right)=\sum_{0 \leq i \leq 2} \rho_{i} \operatorname{Tr}\left(v_{i} v_{i}^{T} \nabla^{2} u(x)\right)=\Delta_{D}^{h} u(x)+\mathcal{O}\left(h^{r}\right),
$$

where again $r=1$ if $x$ is adjacent to $\partial \Omega$, and $r=2$ otherwise.

We illustrate on Figure (1) the relation between the anisotropy defined by a symmetric positive definite matrix $D \in S_{2}^{++}$, and the corresponding offsets $\pm v_{0}, \pm v_{1}, \pm v_{2} \in \mathbb{Z}^{2}$ in Selling's formula. (The weights $\rho_{i}$ are illustrated on Figure 2.) For that purpose, we rely on a parametrization $\mathbf{D}$ of the $2 \times 2$ symmetric positive definite matrices of unit trace, by the points $(x, y)$ of the open unit ball:

$$
\mathbf{D}(x, y):=\frac{1}{2}\left(\begin{array}{cc}
1+x & y \\
y & 1-x
\end{array}\right), \quad \text { where } x^{2}+y^{2}<1 .
$$

This parametrization is closely related to the Pauli matrices in quantum mechanics. A $\mathbf{D}(x, y)$-obtuse superbase is known explicitly, depending on a suitable triangulation of the unit disc, see Figure (1, right).

Definition 2.3 (Discrete degenerate ellipticity Obe06]). A numerical scheme on a finite set $X$ is a map $F: U \rightarrow U$, where $U:=\mathbb{R}^{X}$ is the set of functions from $X$ to $\mathbb{R}$, of the form:

$$
F u(x):=F\left(x, u(x),(u(x)-u(y))_{y \in X \backslash\{x\}}\right),
$$

for all $u \in U, x \in X$. It is Discrete Degenerate Elliptic (DDE) iff $F$ is nondecreasing w.r.t. the second argument $u(x)$, and w.r.t. each $u(x)-u(y), y \in X \backslash\{x\}$. 


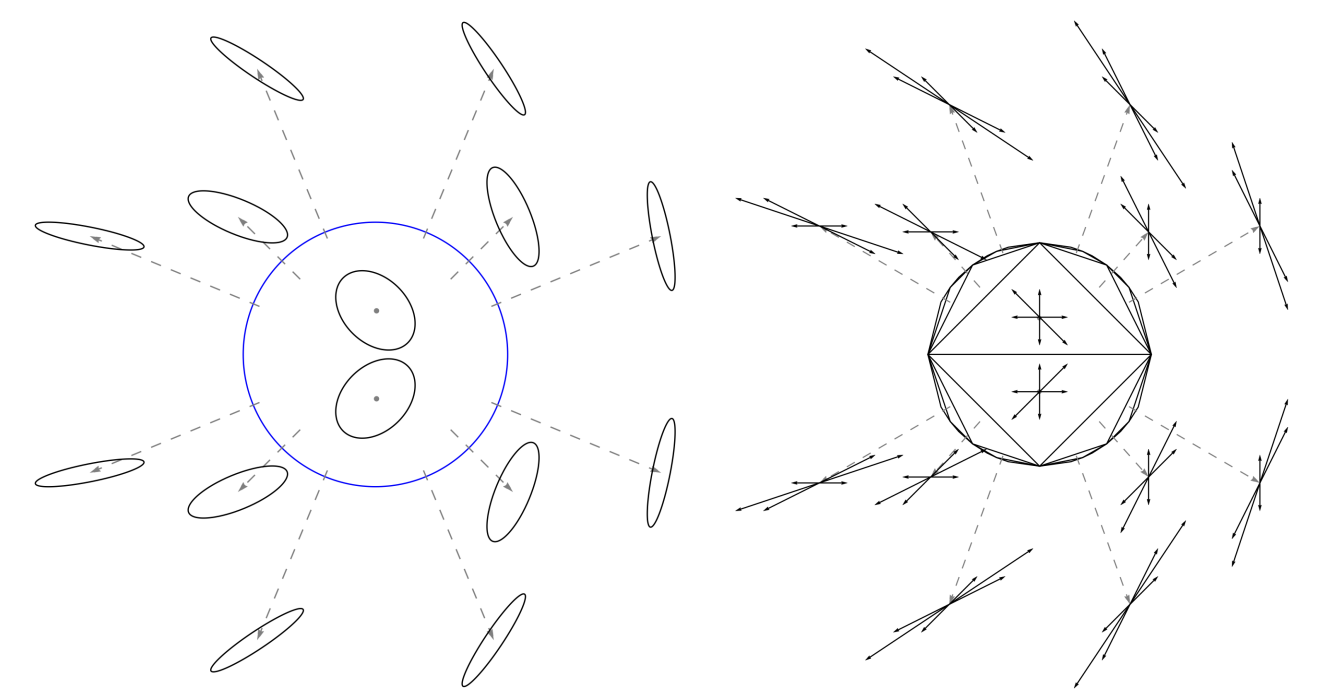

Figure 1: (Left) Ellipsoid $\left\{v \in \mathbb{R}^{2} ; v^{T} \mathbf{D}(z) v=1\right\}$ for some points $z$ of the unit disc, see (9). Anisotropy degenerates as $z$ moves toward the unit circle, shown blue. (Right) $\mathbf{D}(z)$-obtuse superbase, and opposites, for the same points $z$. This superbase is piecewise constant on an infinite triangulation of the unit disk [Sch09], shown black.

Notation: the expression $F u(x)$ should only be regarded as a shorthand for the accurate yet more verbose (10, right). In our application $X:=\Omega_{h}$.

The numerical scheme $-\Delta_{h}^{D}$ is DDE on $\Omega_{h}$, thanks to the non-negativity of the weights $\left(\rho_{i}\right)_{0 \leq i \leq 2}$, and to the finite differences expression (4, right) and (5), where $u$ is extended to $\partial \Omega$ with the provided Dirichlet boundary values. On this basis we obtain a DDE discretization of nonlinear second order operators in Bellman form (1)

$$
\Lambda_{h} u(x):=\sup _{\alpha \in \mathcal{A}} a_{\alpha}+b_{\alpha} u(x)-\Delta_{h}^{D_{\alpha}} u(x), \quad \Lambda_{h} u(x)=\Lambda u(x)+\mathcal{O}\left(h^{r}\right),
$$

where again $r=1$ if $x$ is adjacent to $\partial \Omega$, and $r=2$ otherwise, at least if $\mathcal{A}$ is compact - which is the case for the Pucci operator. As shown in the next section, the supremum in (11, left) can be computed analytically in closed form, for the Pucci PDE, so that the numerical scheme $\Lambda_{h}$ is explicit in terms of the unknown $u$.

Efficient construction of the Jacobian matrix of the numerical scheme. We use a Newton method to solve the discretized PDE, which requires assembling the sparse Jacobian matrix of the numerical scheme (11). In order to describe this essential step, let us rewrite the scheme in the following form (omitting the scale $h$ for readability)

$$
\max _{\alpha \in \mathcal{A}} \mathcal{F}\left(\alpha, x, u(x),\left(u(x)-u\left(y_{1}(x)\right)\right)_{i=1}^{I}\right)=0 .
$$

In comparison with (10), the expression (12) emphasizes (i) that $F$ is defined as a maximum over a parameter set $\mathcal{A}$, and (ii) that the active stencil $y_{1}(x), \cdots, y_{I}(x)$ of a point $x \in \Omega_{h}$ only involves a small number of neighbors. The Jacobian matrix construction, at a given $u: \Omega_{h} \rightarrow \mathbb{R}$, involves the following steps:

1. Compute the maximizer $\alpha^{*}(x)$ in $(12)$, for each $x \in \Omega_{h}$. 


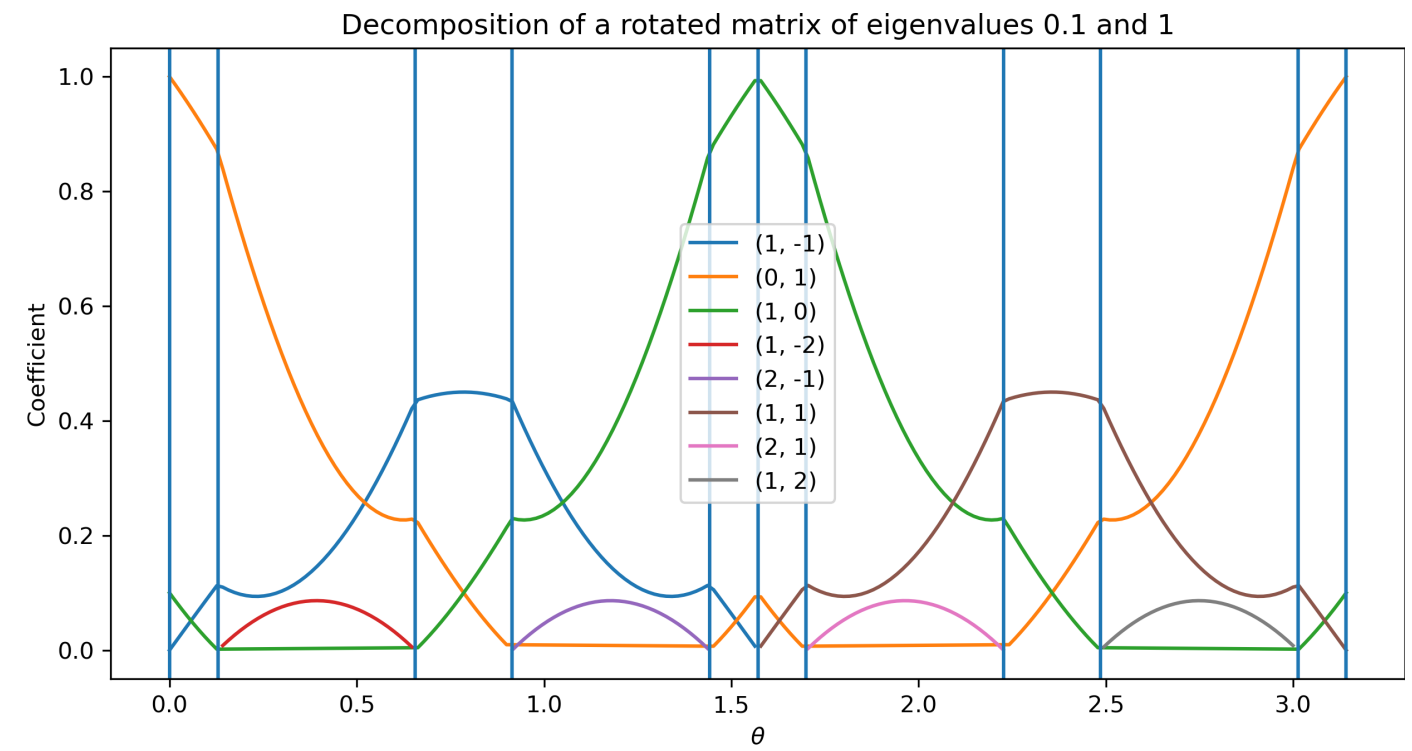

Figure 2: Coefficients of Selling's decomposition (7) of the matrix $D(\theta, \mu)$ for $\theta \in[0, \pi]$ and $\mu=0.1$, see (13). The vertical bars correspond to the angles $0=\theta_{0}<\cdots<\theta_{N}=\pi$ where the support $e_{0}, e_{1}, e_{2} \in \mathbb{Z}^{2}$ of the decomposition changes, see legend.

2. Differentiate the function $\mathcal{F}\left(\alpha^{*}(x), x, \delta,\left(\eta_{i}\right)_{i=1}^{I}\right)$ w.r.t. parameters $\delta$ and $\eta_{1}, \cdots, \eta_{I}$, at the values $u(x)$ and $u(x)-u\left(y_{i}(x)\right), 1 \leq i \leq I$, respectively.

3. Fill the corresponding entries of the sparse Jacobian matrix. More precisely, omitting the arguments of $\mathcal{F}$ for readability

$$
J_{x, x}=\frac{\partial \mathcal{F}}{\partial \delta}+\sum_{1 \leq i \leq I} \frac{\partial \mathcal{F}}{\partial \eta_{i}}, \quad J_{x, y_{i}(x)}=-\frac{\partial \mathcal{F}}{\partial \eta_{i}}, 1 \leq i \leq I .
$$

A custom automatic differentiation toolbox, open source and developed by the third author, makes these operations transparent. The above computations rely on the envelope theorem [Car01, which states that the value function to an optimization problem, here 112 , over a compact set, here $\mathcal{A}$, is differentiable w.r.t. the parameters, here $\delta$ and $\left(\eta_{i}\right)_{i=1}^{I}$, whenever the problem solution, here $\alpha^{*}(x)$, is single valued (which is a generic property). In addition the first order derivatives have the expression used above, obtained by freezing the optimization parameter $\alpha \in \mathcal{A}$ to the optimal value $\alpha^{*}(x)$.

\subsection{The Pucci operator}

The Bellman form of the Pucci operator (3) involves a family of matrices $D(\theta, \mu)$, parameterized by the inverse $0<\mu \leq 1$ of their condition number, and by an angle $0 \leq \theta \leq \pi$. As a starter, we rewrite those in the form (9)

$$
D(\theta, \mu)=(1+\mu) \mathbf{D}\left(\frac{1-\mu}{1+\mu} \mathrm{n}(2 \theta)\right)
$$

where $\mathrm{n}(\varphi):=(\cos \varphi, \sin \varphi)$. Note that the argument of $\mathbf{D}$ in $(9)$ describes a circle of fixed radius $\frac{1-\mu}{1+\mu}$ within the unit disc, see Figure 1. Thus one can find $0=\theta_{0}<$ 
$\cdots<\theta_{N}=\pi$, where $N=N(\mu)$, such that on each interval $\left[\theta_{n}, \theta_{n+1}\right]$ the superbase $\left(e_{0}^{n}, e_{1}^{n}, e_{2}^{n}\right)$ is $D(\theta, \mu)$-obtuse and the coefficients in (7) take the form

$$
\rho_{i}(\theta)=-\left\langle e_{i-1}^{n}, D(\theta, \mu) e_{i+1}^{n}\right\rangle=\alpha_{i}^{n}+\beta_{i}^{n} \cos (2 \theta)+\gamma_{i}^{n} \sin (2 \theta),
$$

for suitable constants $\alpha_{i}^{n}, \beta_{i}^{n}, \gamma_{i}^{n} \in \mathbb{R}, 0 \leq i \leq 2,0 \leq n<N$, see Figure 2, One finds that $N(1 / 4)=2, N(1 / 10)=10, N(1 / 400)=122$, and one can show that $N(\mu) \leq C \mu^{-1}|\ln \mu|$ for some constant $C$ independent of $\mu$. By linearity of (8) one also has

$$
\Delta_{h}^{D(\theta, \mu)} u(x)=\alpha^{n}+\beta^{n} \cos (2 \theta)+\gamma^{n} \sin (2 \theta)
$$

for all $\theta \in\left[\theta_{n}, \theta_{n+1}\right]$, whose coefficients $\alpha^{n}, \beta^{n}, \gamma^{n}$ depend on $\rho, h, u$ and $x$. Therefore, evaluating the discretized Bellman operator (11) associated with the Pucci equation (3) at a point $x \in \Omega_{h}$ amounts to solving a small number $N$ of optimization problems, whose value is explicit. These optimization problems, and their value, take the following generic form

$$
\begin{aligned}
& \max _{\varphi \in\left[\varphi_{*}, \varphi^{*}\right]} \alpha+\beta \cos \varphi+\gamma \sin \varphi \\
& =\left\{\begin{array}{lr}
\alpha+\sqrt{\beta^{2}+\gamma^{2}} & \text { if } \arg (\beta+i \gamma) \in] \varphi_{*}, \varphi^{*}[, \\
\alpha+\max \left\{\beta \cos \varphi_{*}+\gamma \sin \varphi_{*}, \beta \cos \varphi^{*}+\gamma \sin \varphi^{*}\right\} & \text { else, }
\end{array}\right.
\end{aligned}
$$

where $\arg (\omega)$ denotes the argument of $\omega \in \mathbb{C}$, taken in $[0,2 \pi[$. In view of $[15]$, we choose $\varphi_{*}=2 \theta_{n}, \varphi^{*}=2 \theta_{n+1}, \alpha=\alpha^{n}, \beta=\beta^{n}$, and $\gamma=\gamma^{n}$. Then, following (3), we take the largest value among $0 \leq n<N$.

\section{$3 \quad$ Numerical experiments}

We present numerical results for the Pucci equation, chosen to illustrate the qualitative behavior of the solutions, and validate the scheme robustness and accuracy on synthetic problems with known solutions. Some of the considered domains are neither smooth nor convex, and the chosen synthetic solutions range from smooth to singular.

The numerical scheme is implemented as described in the previous section, and a Newton method is used to solve the resulting coupled systems of non-linear equations. In practice, convergence to machine precision is achieved in a dozen of iterations, without damping, from an arbitrary guess. An open source Python $\AA$ notebook reproducing (most of) the illustrations of this paper is available on the third author's webpag $\AA^{1}$.

We illustrate on Figure 3 the transition of the Pucci equation from a strongly elliptic Laplacian-like PDE to a combinatorial-type convex-envelope problem, as the parameter $\mu$ takes values $1 / 4$ and $1 / 400$. The chosen domain is non-smooth and non-simply connected : $\Omega:=U \backslash U^{\prime}$ where $U:=B(0,1) \cup(] 0,1[\times]-1,1[)$ and $U^{\prime}:=k R_{\theta}(U)$ is its image under a scaling $(k=0.4)$ and a rotation $(\theta=\pi / 3)$. The boundary condition is 1 on $\partial U$, and 0 on $\partial U^{\prime}$, and the r.h.s is $f \equiv 0$. The discretization grid size is $100 \times 100$, and the computation time is $1 s$ for $\mu=1 / 4$, and $45 s$ for $\mu=1 / 400$. The time difference is attributable to the complexity of the

\footnotetext{
${ }^{1}$ Link : Github.com/Mirebeau/AdaptiveGridDiscretizations, see chapter 2.B.III.
} 
numerical scheme, which involves $N=2$ pieces for in the first case and $N=122$ in the latter, due to the larger condition number of the diffusion tensors $D(\theta, \mu)$, see $\$ 2.2$ Nevertheless, the number $N=N(\mu)$ is independent of the grid scale, and both schemes are second order consistent. In the case $\mu=1 / 400$, the PDE solution is quite close to the convex envelope of the boundary conditions, whose gradient is constant in some regions, and discontinuous across some lines, see Figure (3, right).

On figure 4, we reconstruct some known synthetic solutions from their image by the Pucci operator, with parameter $\mu=0.2$, and their trace on the boundary. The examples are taken from the literature [FJ17, FO13], and the reconstruction errors are provided in the $L^{1}$ and $L^{\infty}$ norm.

- $($ Smooth example [FJ17] $) u(x)=\left(x^{2}+y^{2}\right)^{2}$ on $\left.\Omega=B(0,1) \cup\right] 0,1\left[^{2}\right.$

- $\left(C^{1}\right.$ example [FO13] $) u(x)=\max \left\{0,\left\|x-x_{0}\right\|^{2}-0.2\right)$ on $\left.\Omega=\right] 0,1\left[^{2}\right.$.

- (Singular example [FO13]) $u(x)=\sqrt{2-\|x\|^{2}}$ on $\left.\Omega=\right] 0,1\left[^{2}\right.$.

Empirically, the $L^{1}$ numerical error behaves like $\mathcal{O}\left(h^{2}\right)$, where $h$ is the grid scale (inverse of resolution in images). The $L^{\infty}$ error behaves like $\mathcal{O}\left(h^{2}\right)$ in the smooth and $C^{1}$ examples, but decays more slowly for the singular solution. Note: we rotated the Cartesian discretization grid by $\pi / 3$ in these experiments, since otherwise the perfect alignment of the domain boundary with the coordinate axes gives an unfair advantage to grid based methods (like ours).

\section{Conclusion}

In this paper, we presented a new strategy for discretizing non-divergence form, fully-nonlinear second order PDEs, and applied it to the Pucci equation. The steps of this approach can be summarized as follows: (i) rewrite the problem in Bellman form, as an extremum of linear equations, (ii) discretize the second order linear operators using monotone finite differences based on Selling's decomposition of positive definite matrices, (iii) solve the pointwise optimization problems involved in the numerical scheme definition, either explicitly (as could be done here), or numerically.

This methodology yields finite difference schemes which are degenerate elliptic, second order consistent, and use stencils of fixed size, in contrast with existing approaches Obe08 which cannot achieve all these desirable properties simultaneously. Numerical experiments confirm that the proposed scheme can extract smooth PDE solutions with second order accuracy, and that it remains stable and convergent for harder problems involving a singularity at a point or along a line. Future research will be devoted to extending the results to other PDEs, such as the Monge-Ampère equation and its variants.

\section{References}

[BCM16] Jean-David Benamou, Francis Collino, and Jean-Marie Mirebeau. Monotone and consistent discretization of the Monge-Ampere operator. Mathematics of computation, 85(302):2743-2775, 2016. 

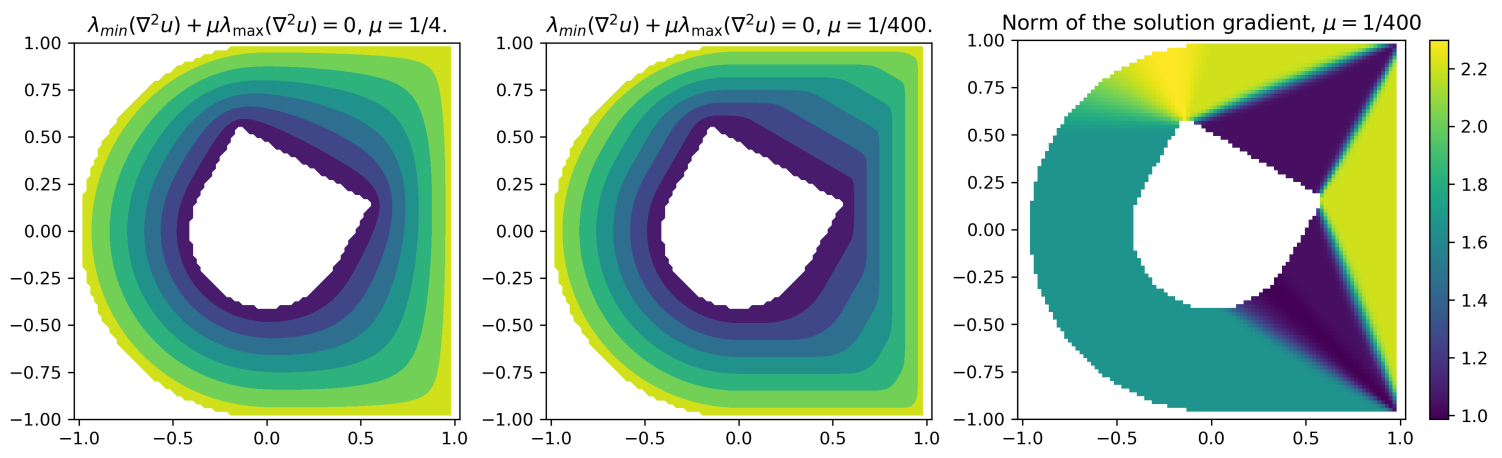

Figure 3: Solution of the Pucci PDE with $\mu=1 / 4$ (left), $\mu=1 / 400$ (center, right: gradient norm)
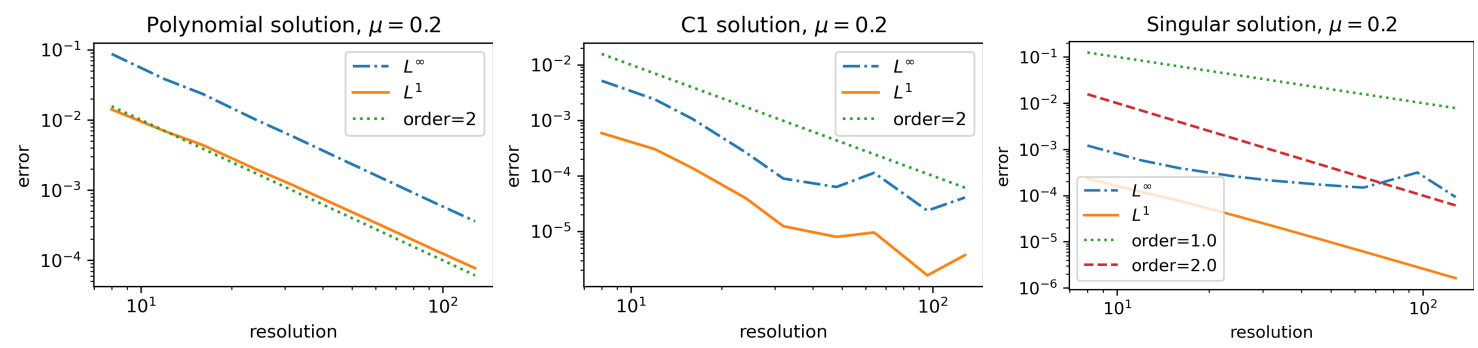

Figure 4: Numerical error as a function of grid size, for synthetic solutions to the Pucci equation.

[BOZ04] J Frédéric Bonnans, Elisabeth Ottenwaelter, and Housnaa Zidani. A fast algorithm for the two dimensional HJB equation of stochastic control. ESAIM: Mathematical Modelling and Numerical Analysis, 38(4):723-735, 2004

[BS91] Guy Barles and Panagiotis E Souganidis. Convergence of approximation schemes for fully nonlinear second order equations. Asymptotic analysis, 4(3):271-283, 1991.

[Car01] Michael Carter. Foundations of mathematical economics. MIT Press, 2001 .

[CIL92] Michael G Crandall, Hitoshi Ishii, and Pierre-Louis Lions. User's guide to viscosity solutions of second order partial differential equations. Bulletin of the American Mathematical Society, 27(1):1-67, 1992.

[FJ17] Xiaobing Feng and Max Jensen. Convergent semi-Lagrangian methods for the Monge-Ampère equation on unstructured grids. SIAM Journal on Numerical Analysis, 55(2):691-712, 2017.

[FM14] Jérôme Fehrenbach and Jean-Marie Mirebeau. Sparse non-negative stencils for anisotropic diffusion. Journal of Mathematical Imaging and Vision, 49(1):123-147, 2014.

[FO13] Brittany D Froese and Adam M Oberman. Convergent Filtered Schemes for the Monge-Ampère Partial Differential Equation. SIAM Journal on Numerical Analysis, 51(1):423-444, January 2013. 
[Mir18] Jean-Marie Mirebeau. Fast-marching methods for curvature penalized shortest paths. Journal of Mathematical Imaging and Vision, 60(6):784$815,2018$.

[Mir19] Jean-Marie Mirebeau. Riemannian Fast-Marching on Cartesian Grids, Using Voronoi's First Reduction of Quadratic Forms. SIAM Journal on Numerical Analysis, 57(6):2608-2655, 2019.

[Obe06] Adam M Oberman. Convergent Difference Schemes for Degenerate Elliptic and Parabolic Equations: Hamilton-Jacobi Equations and Free Boundary Problems. SIAM Journal on Numerical Analysis, 44(2):879-895, January 2006.

[Obe08] Adam M Oberman. Wide stencil finite difference schemes for the elliptic Monge-Ampere equation and functions of the eigenvalues of the Hessian. Discrete Contin Dyn Syst Ser B, 2008.

[Sch09] Achill Schürmann. Computational geometry of positive definite quadratic forms. University Lecture Series, 49, 2009.

[Sel74] Eduard Selling. Ueber die binären und ternären quadratischen Formen. Journal fur die Reine und Angewandte Mathematik, 77:143-229, 1874. 